\title{
A short review of history and technical advances in quantitative neuromuscular monitoring devices
}

\author{
Wonjin Lee ${ }^{1}$, Ki Tae Jung ${ }^{2}$ \\ ${ }^{1}$ Department of Anesthesiology and Pain Medicine, Busan Paik Hospital, Paik Institute for Clinical Research, Inje University College \\ of Medicine, Busan, ${ }^{2}$ Department of Anesthesiology and Pain Medicine, Chosun University Hospital, School of Medicine and Medical \\ College, Chosun University, Gwangju, Korea
}

Received June 4, 2021

Revised June 15, 2021

Accepted June 16, 2021

Corresponding author

Ki Tae Jung

Department of Anesthesiology and

Pain Medicine, Chosun University

Hospital, 365 Pilmun-dearo, Dong-

gu, Gwangju 61453, Korea

Tel: +82-62-220-3223

Fax: +82-62-223-2333

E-mail: mdmole@chosun.ac.kr

ORCID:

https://orcid.org/0000-0002-2486-9961
Anesthetic procedures using a neuromuscular blocking drug (NMBD) always have a potential risk of residual neuromuscular block (RNMB) associated with serious respiratory complications despite the use of reversal agents. Due to the risk of RNMB, neuromuscular monitoring is a very important tool for assessing patient safety. Recently, the importance of quantitative neuromuscular monitoring has been highlighted because of the advent of sugammadex, which determines dosage based on the degree of NMB. Neuromuscular monitoring, which has been used clinically since the 1970s, is an appropriate method for assessing the level of neuromuscular blockade (NMB) after the use of a NMBD during anesthesia. Although the basic principles have not changed considerably, recent technical advances in neuromuscular monitoring devices can help anesthesiologists with convenient quantitative neuromuscular monitoring to accurately evaluate the levels of NMB, choose an appropriate NMBD, and avoid RNMB. The recently released electromyography-based devices provide more accurate values than acceleromyography (AMG). Although AMG has a problem with overestimation, modern AMG devices with new three-dimensional technology overcome this limitation. Anesthesiologists should increase their knowledge of neuromuscular mechanisms and monitoring, including how to use the latest device for proper neuromuscular monitoring and patient safety. In this review, we have explored the short history of neuromuscular monitoring devices and the latest trends in technology development.

Keywords: Delayed emergence from anesthesia; Electromyography; Neuromuscular blockade; Neuromuscular blocking agents; Neuromuscular monitoring

\section{INTRODUCTION}

Neuromuscular blocking drugs (NMBDs), which are used for not only endotracheal intubation during the induction of anesthesia but also the maintenance of anesthesia are inextricably linked to the anesthesiologist. However, anesthetic procedures using a NMBD always have a potential risk of residual neuromuscular block (RNMB), which is associated with serious respiratory complications such as airway obstruction, hypoxia, and aspiration pneumonia, despite the use of reversal agents in the operating room [1]. Due to the risk of RNMB, neuromuscular monitoring is a very important tool for assessing patient safety. Although neuromuscular monitoring is an appropriate method for assessing the level of neuromuscular blockade (NMB) after the use of a NMBD during anesthesia [1], it is paradoxical that many anesthesiologists do not monitor neuromuscular function or make accurate judgments of the data obtained from 
quantitative neuromuscular monitoring despite knowing the importance of neuromuscular monitoring [2,3]. In Korea, perioperative neuromuscular monitoring was included as an anesthesia adequacy assessment in 2018, and it is expected that the awareness of neuromuscular monitoring and its actual use will increase.

Neuromuscular monitoring devices have been in clinical use since the 1970s [4], and the basic principles have not changed considerably. However, with the recent development of technology, many neuromuscular monitoring devices with increased convenience and accuracy have been developed. In this review, we have explored the short history of neuromuscular monitoring devices and the latest trends in technology development.

\section{SHORT HISTORY OF TECHNICAL DEVELOPMENT OF NEUROMUSCULAR MONITORING DEVICES}

The history of technological advances in the field of neuromuscular monitoring devices is very interesting [5]. After Harold Griffiths introduced the use of curare for abdominal surgery in 1942, the use of NMBDs became widespread in surgery in the 1950s. However, Beecher and Todd reported an increase in mortality after anesthesia in patients using d-tubocurarine in 1954, which led physicians to misunderstand that NMBDs are toxic [6]. Dripps et al. [7] observed that the increase in mortality after anesthesia was attributed to comorbidities in patients and not the NMBD toxicity. Thus, there is a consensus about the need for safety in the use of NMBDs.

In 1952, Thesleff conducted a study on muscle tension during administration of succinylcholine using myography, which recorded the flexion twitches of ulnar fingers during the stimulation of the ulnar nerve at the elbow [8]. A clinical neuromuscular monitor for anesthesiologists was developed by Christie and Churchill-Davidson in 1958 [9]. The monitor called "St Thomas' Hospital Nerve Stimulator was aimed to discriminate between residual paralysis and narcotization conveniently by observing the response of the adductor pollicis (AP) muscle after ulnar nerve stimulation at the wrist. They also developed a new nerve stimulator that can apply both twitch and tetanic stimulation in 1965 [10].

Although Churchill-Davidson described a method similar to train-of-four (TOF) in 1965, the TOF ratio was first used for patients with myasthenia gravis in 1968. Roberts and Wilson reported the fade phenomenon during TOF stimulation in patients with myasthenia gravis and suggested the use of the TOF ratio for the assessment of treatment effects [11]. The use of TOF under anesthesia was introduced by Ali et al. in 1970 [12]. Their study demonstrated the absence of fade after the use of a depolarizing NMBD and forecasted the usefulness of TOF monitoring in the assessment of the degree of NMB. They introduced the TOF ratio as an indicator of the degree of $\mathrm{NMB}$ and reported that recovery from NMB can be achieved with a TOF ratio above 0.6, which is comparable to the ability to lift the head for 5 seconds $[13,14]$. A modern nerve stimulator with a single twitch, TOF, TOF ratio, and post-tetanic count (PTC) was designed and introduced by Viby-Mogensen et al. in 1980 [15]. Since then, many neuromuscular monitoring devices have been developed and used, and anesthesiologists' interest in neuromuscular monitoring has also increased.

\section{DEGREES OF NEUROMUSCULAR BLOCKADE}

Although there is a consensus on the definition of the exact depth of NMB, modified degrees of NMB have been suggested in the international consensus conference [16-18]. After administration of the intubation dose of a NMBD, a patient rapidly progresses to a state of intense block, and the response of TOF and PTC disappears (Fig. 1). Deep block, the phase after intense block, is defined as the phase when the response to tetanic stimulation begins. After achieving a PTC of $>6-8$, the recovery to TOF count $=1$ is expected soon, indicating a phase of the moderate block [16]. Moderate block is defined as the phase when 1-3 TOF twitch appears. After the appearance of the fourth twitch of TOF and it possible to calculate the TOF ratio, the recovery phase begins. The recovery phase can be categorized as a light (shallow) block, minimal block (near recovery), and full recovery according to the TOF ratio. In light block (TOF ratio: 0.1-0.4), fade can be observed during subjective TOF monitoring; however, fade disappears during minimal block (TOF ratio $>0.4$ to $<0.9$ ) and full recovery (TOF ratio $\geq 0.9$ ).

Recently, Biro et al. [19] has suggested modified degrees of neuromuscular block and separated a broad degree of 


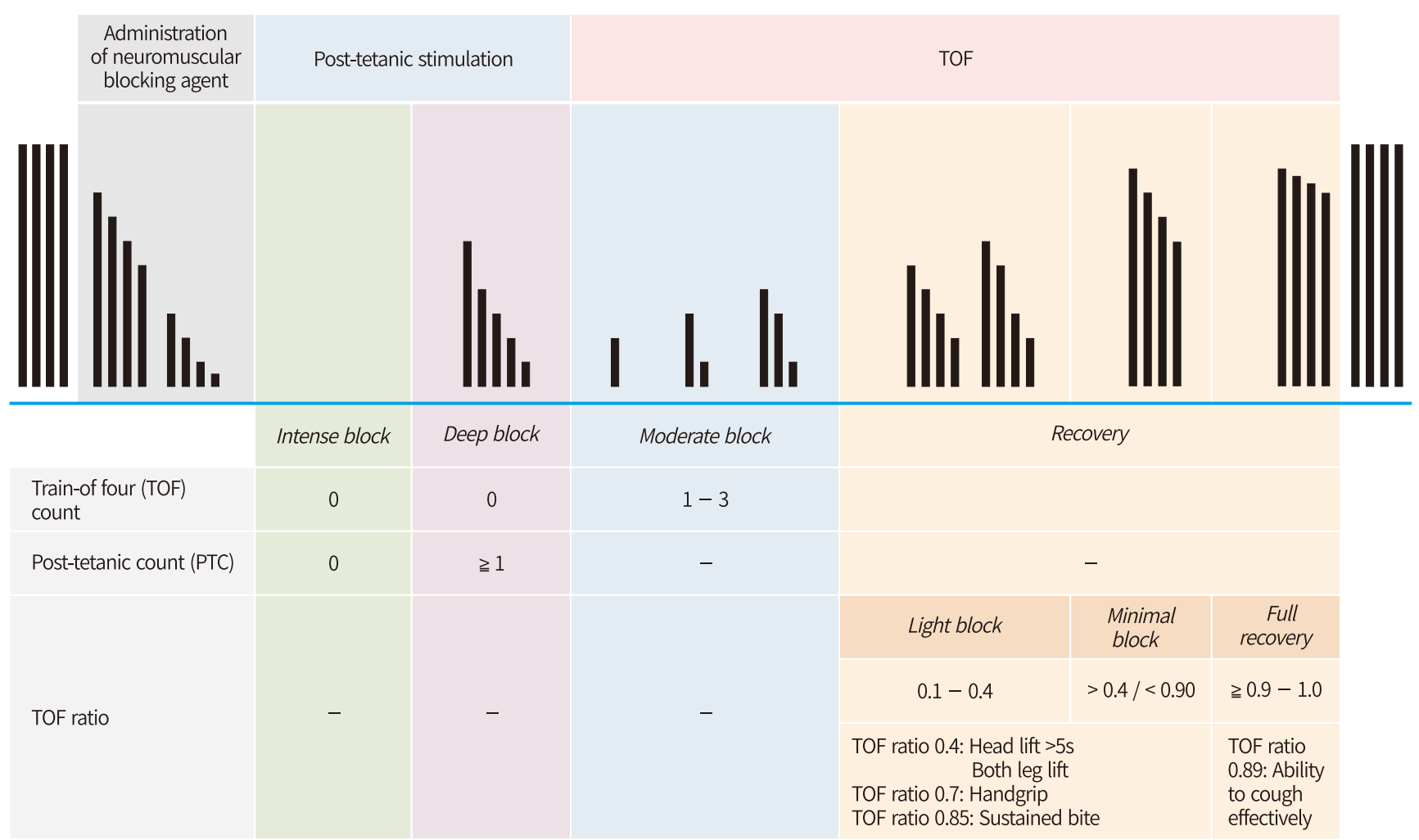

Fig. 1. Suggested definitions of degrees of neuromuscular block [16].

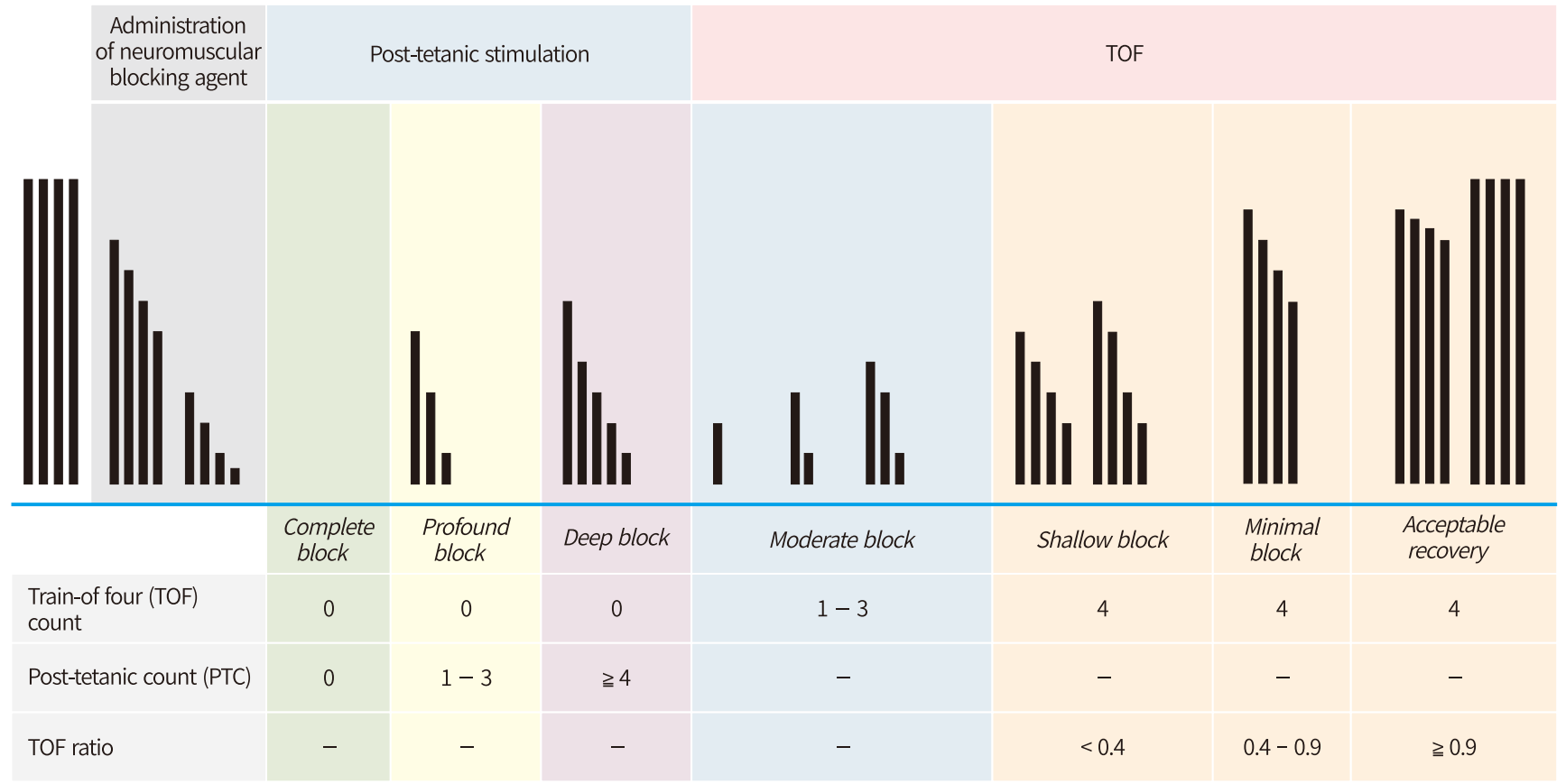

Fig. 2. Recently proposed modified degree of neuromuscular block [19].

deep block (from TOF count $=0$ to $P T C ~ \geq 1$ ) into profound block (from TOF count=0 to PTC 1-3) and deep block (PTC 24) for clinical application (Fig. 2). For example, some surgeries such as laparoscopic, robotic, eye, and airway surgery require profound block (more intense than deep block) to prevent any movement. Moreover, it may take a shorter 
time to close the wound in such surgeries than in traditional surgical procedures, and conventional reversal agents such as neostigmine or pyridostigmine would not be effective for the reversal of NMB in the state of profound block. Therefore, a detailed classification may be necessary for the selection of reversal agents and avoid the risk of RNMB.

\section{MODALITIES OF NEUROMUSCULAR MONITORING}

Several modes of neuromuscular monitoring have been introduced to assess and monitor NMB (Table 1). Mechanomyography (MMG), which measures the mechanical response of the adductor pollicis muscle induced by ulnar nerve stimulation, is the gold standard for neuromuscular monitoring because of its precise results and reproducibility [1]. However, it is not used in clinical situations because of the difficulty involved in its setup. Clinically, acceleromyography (AMG) and electromyography (EMG) are commonly used for the quantitative measurement of NMB by mechanical or electrical response [17]. AMG measures the force by the acceleration movement of muscles, such as the flexor hallucis brevis, orbicularis oculi, or corrugator supercilia, using Newton's second law of motion (force=mass acceleration). EMG measures the evoked action potential of target muscles (e.g., the adductor pollicis, abductor digiti minimi, or first dorsal interosseus muscles) during muscle contraction, which is produced by the stimulation of peripheral nerves (e.g., the ulnar nerve) via skin electrodes. Other modalities such as kinemyography (KMG), which measures the voltages during the bending of a piezoelectric sensor strip, and phonomyography (PMG), which measures the low-fre- quency sounds evoked by muscle contraction are also used for the assessment of neuromuscular function.

\section{AMG VS. EMG}

AMG is the most widely used modality owing to its advantages, which produces a real-time measurement of objective neuromuscular function at a low cost. However, there are several limitations to its clinical use. AMG requires calibration before the first injection of a NMBD to detect the supramaximal current for the adjustment of twitch response corresponding to $100 \%$. Moreover, maintaining the arm posture and measurement environments, such as restriction of arm and thumb movement throughout the surgery and avoidance of impediment by drapes, are required throughout AMG measurement because the acceleration sensor of AMG measures only in one plane of motion. Thus, AMG cannot be applied in surgery, which interferes with the position of the arm that measures the NMB, unless a special protective device is used. However, a modern AMG device with three-dimensional sensor technology has been introduced to overcome the drawbacks of calibration and postural limitations by assessing the complex motion of the thumb in response to neurostimulation. AMG monitors with a three-dimensional sensor can be applied to surgeries that involve tucking arms close to the body. AMG also has a problem called the "reverse fade effect, showing a TOF ratio higher than the ideal baseline value of 1.0. Suzuki et al. [20] have reported a TOF ratio of $1.10-1.47$. This can lead to problems such as the overestimation of the TOF ratio during recovery from NMB.

EMG measures the action potential and converts it into

Table 1. Modalities for neuromuscular monitoring

\begin{tabular}{|c|c|c|c|}
\hline Modes & Measures & Sensor & Note \\
\hline Mechanomyography & Force of contraction & Force & $\begin{array}{l}\text { Gold standard with precise and reproducible result } \\
\text { Difficult to adapt clinically due to difficulty of setup } \\
\text { the equipment }\end{array}$ \\
\hline Acceleromyography & $\begin{array}{l}\text { Force by the acceleration movement of } \\
\text { muscle }\end{array}$ & Piezoelectric crystal & $\begin{array}{l}\text { Most used } \\
\text { Reliable train-of-four ratio } \\
\text { Overestimation }\end{array}$ \\
\hline Electromyography & Amplitude of action potential of muscle & Electrodes & $\begin{array}{l}\text { Best indicator of pure neuromuscular function } \\
\text { Affected by electrocautery or temperature }\end{array}$ \\
\hline Kinemyography & $\begin{array}{l}\text { Voltage generated during the movement of } \\
\text { muscle }\end{array}$ & Piezoelectric sensor & Less reproducible than electromyography \\
\hline Phonomyography & Sound intensity & \multicolumn{2}{|c|}{ High-fidelity microphone Low clinical use } \\
\hline
\end{tabular}


a mechanical response. It can be the best indicator of pure neuromuscular function because of low interference with other events and stable amplitude despite constant stimulation [1]. EMG physiologically measures neuromuscular function using electrical signals rather than the force of muscle contraction, which provides more precise results than AMG. The neuromuscular function data obtained from EMG are most similar to those obtained from MMG [21]. Moreover, unlike AMG, it does not require a special setup or care for position and does not have the reverse fade effect. Although EMG has the possibility of incorrect measurement due to interference from electrical stimulation such as electrocautery, modern EMG has an automatic function of pausing and resuming the measurement by detecting electrical interference. Another minor disadvantage of EMG is its higher cost than AMG because of the use of a unique electrode in EMG devices.

The overestimation of the TOF ratio by AMG can be problematic for the goal of RNMB avoidance as it leads to the misconception that recovery from NMB is achieved with a TOF ratio value of $\geq 0.9$, although the normalized value is $<0.9$. According to previous studies, the TOF ratio obtained from AMG was $10 \%-20 \%$ higher than that obtained from MMG or EMG [20,22,23]. Kopman et al. [24] reported that the differences in the TOF ratio between EMG and AMG when the TOF value of AMG had recovered to approximately 0.7 and 0.9 were $0.069-0.125$ and $0.055-0.096$, respectively (Fig. 3). Although the application of an elastic preload

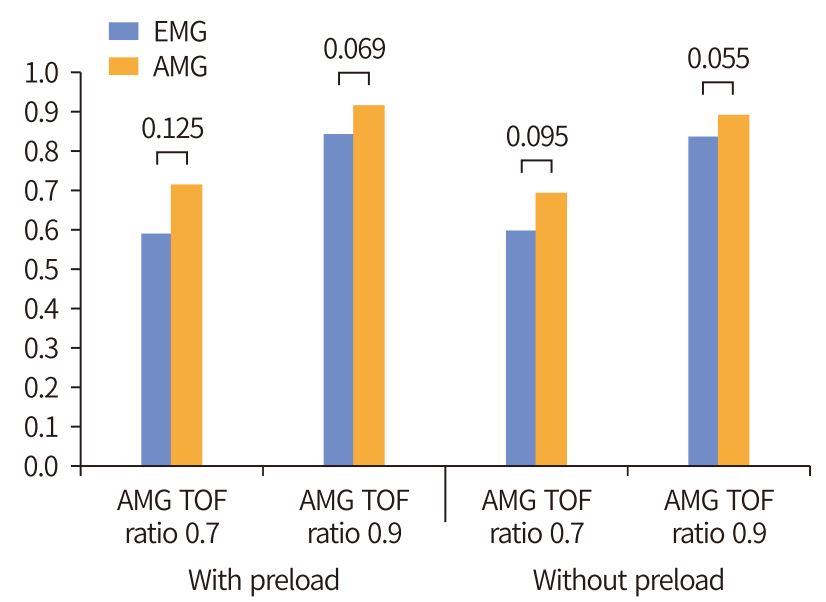

Fig. 3. Difference in the TOF ratio between EMG and AMG during recovery from NMB [24]. The of the TOF ratio of EMG at both TOF ratios of AMG of 0.70 and 0.90 shows smaller values. TOF: train-of-four, EMG: electromyography, AMG: acceleromyography. to the thumb decreased the variability of baseline TOF values, the difference in the TOF ratio between EMG and AMG increased during recovery. They concluded that the TOF values of AMG overestimated the degree of recovery from NMB compared to the TOF values of EMG, and TOF values $<0.90$ obtained from AMG are inaccurate and indicate incomplete recovery from NMB. Furthermore, "normalization of each measured value of the TOF ratio is difficult and requires more time to interpret. Therefore, a simple method of reducing the TOF ratio by approximately $10 \%$ can be used or a method aiming at 1.0 instead of 0.9 as a target value for the recovery from NMB in AMG can be used, but this is an inaccurate measurement [25].

\section{MODERN TECHNICAL DEVELOPMENT OF NEUROMUSCULAR MONITORING DEVICES}

With the recent development of technology, neuromuscular monitoring devices that are close to the ideal are being released. An ideal neuromuscular monitoring device requires functions such as large monitors for the trends display and annotation of the events, short TOF intervals (approximately 20 seconds), a warning system with user setting threshold limits, and automatic PTC mode [16]. Recently introduced neuromuscular monitoring devices such as the TOFscan (IdMed, Marseille, France), TetraGraph (Senzime BV, Uppsala, Sweden), and TwitchView (Blink Device Company, Seattle, USA) can implement most of these functions (Table 2).

In particular, the automatic function called automatic PTC or automatic TOF-PTC mode is very useful for anesthesiologists during the induction and maintenance of anesthesia (Fig. 4) [16]. After starting neuromuscular monitoring with automatic mode and administration of a NMBD, the device would measure TOF in short intervals (20-30-second interval) repeatedly if the TOF count appears. When the TOF count becomes 0 , the PTC mode starts for the assessment of deep or profound blocks with approximately 3-5-minute interval to avoid inaccurate measurement by post-tetanic potentiation. The measurement of PTC is automatically repeated until the TOF count becomes 1 . Then, the PTC sequence is finished and reverted to the TOF mode with short intervals automatically. Among the new neuromuscular monitors available in Korea, TOFscan and TwitchView provide the automatic PTC mode. 
Table 2. Characteristics of up-to-date neuromuscular monitoring devices available in Korea and the comparison with TOF Watch ${ }^{\circledR}$ SX

\begin{tabular}{|c|c|c|c|c|}
\hline & TOF Watch $^{\circledast}$ SX & TOFscan $^{\circledast}$ & Tetragraph $^{\circledR}$ & Twitch View ${ }^{\circledR}$ \\
\hline Type & AMG & AMG & EMG & EMG \\
\hline Pulse & Monophasic /200, $300 \mu \mathrm{s}$ & Monophasic /200 $\mu \mathrm{s}$ & Monophasic /200, $300 \mu \mathrm{s}$ & Monophasic /100, 200, $300 \mu \mathrm{s}$ \\
\hline Current (Automatic calibration) & Automatic & 40 mA (20-60 mA) & Automatic (10-60 mA) & Automatic $(0-80 \mathrm{~mA})$ \\
\hline Automatic TOF mode & - & $15,30 \mathrm{~s}, 1 \mathrm{~m}, 2 \mathrm{~m}, 5 \mathrm{~m}, 15 \mathrm{~m}$ & $20 \mathrm{~s}, 1 \mathrm{~m}, 5 \mathrm{~m}, 15 \mathrm{~m}, 60 \mathrm{~m}$ & $10 \mathrm{~s}, 2 \mathrm{~m}, 5 \mathrm{~m}, 15 \mathrm{~m}, 60 \mathrm{~m}$ \\
\hline PTC mode & + & Lockout about 2 m $30 \mathrm{~s}$ & + & Lockout about $5 \mathrm{~m}$ \\
\hline Automatic PTC mode & - & + & - & + \\
\hline DBS mode & + & $3.3,3.2$ & - & - \\
\hline ST mode & - & $0.1,1 \mathrm{~Hz}$ & $1 \mathrm{~Hz}$ & + \\
\hline TET mode & $5 s-50$ or $100 \mathrm{~Hz}$ & $50 \mathrm{~Hz}$ & - & + \\
\hline Battery & 9 V Alkaline & 2,000 mA Li-lon & $8 \mathrm{hrs}$ & $8 \mathrm{hrs}$ \\
\hline Sensor & Reusable & Reusable/Disposable & Disposable & Disposable \\
\hline Simulator & - & - & + & - \\
\hline
\end{tabular}

AMG: acceleromyography, EMG: electromyography, TOF: train-of-four, PTC: post-tetanic count, DBS: double burst suppression, ST: single twitch, TET: tetanic stimulation.

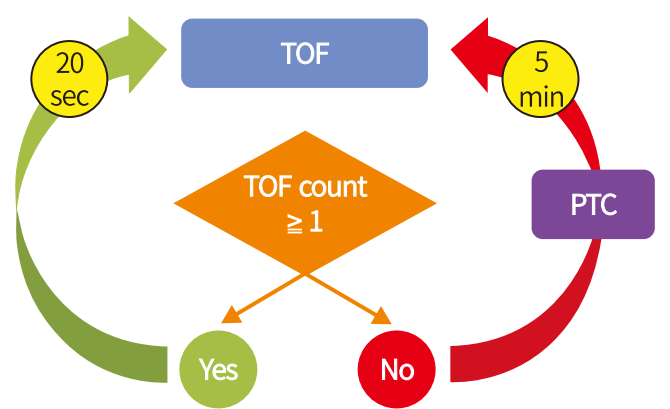

Fig. 4. Schematic algorithm of automatic PTC mode. Measurement of TOF with 20-30-second interval repeats until the disappearance of TOF count and the PTC mode starts with about 3-5-minute interval automatically for the assessment of deep or profound block. When the TOF count reappears, PTC mode is terminated automatically and reverted to the TOF mode. TOF: train-of-four, PTC: post-tetanic count.

However, some neuromuscular monitors have not been developed in Korea. StimPod NMS450 (Xavant Technology Ltd., South Africa) is an interesting device that can use both AMG with triaxial accelerometer and EMG. However, the TOF count of AMG was approximately 39\% smaller than that of EMG, which represents the underestimation of the TOF count in the AMG with the StimPod [26]. TOF-cuff (RGB Medical, Madrid, Spain) has a unique mechanism using a compressomyography technique that measures TOF responses by changes in pressure peaks during muscle contraction induced by stimulation of the brachial plexus [16]. TOF-cuff has the advantage of convenience because it uses a modified blood pressure cuff on the arm for neuromuscular monitoring, but has limitations of underestimation in the measurement of TOF ratios and inaccuracy for the prevention of RNMB in comparison to the EMG or AMG [27]. Recent studies showed delayed recovery to a normalized TOF ratio of $>0.9$, approximately 25 -minute longer, with EMG or AMG compared with TOF-Cuff [28].

\section{CONCLUSION}

Many experts have emphasized the importance of objective quantitative neuromuscular monitoring, and consensus on the imperatives of neuromuscular monitoring during the use of NMBDs has been achieved in several guidelines [16,18,29-32]. However, a large number of patients (40\%$60 \%$ leave the operating room without an acceptable recovery from NMB, and they are exposed to the potential risk of complications associated with RNMB [31,33,34]. In particular, qualitative peripheral nerve stimulation in the decision to recover from NMB creates RNMB [31]. Furthermore, the importance of quantitative neuromuscular monitoring has been highlighted with the advent of sugammadex, which requires the determination of dosage based on the degree of NMB [32]. In addition, with the recent increase in the number of surgeries requiring deep or profound NMB, which is almost impossible to guarantee full recovery with the use of conventional reverse agents, the need for neuromuscular monitoring is increasing. Thus, objective quantitative neuromuscular monitoring has become mandatory for the management of anesthetized patients after the use of NMBDs in 
recent years.

Modern neuromuscular monitors with a state-of-the-art system make it easier and more accurate than ever to evaluate neuromuscular functions during anesthesia. Although AMG is the most commonly used device, it has positional and overestimation limitations. However, AMG devices with modern 3D technology overcome the previous limitations if used properly and provide ease of use and acceptable results. The recently released EMG-based devices are convenient with disposable sensors. Moreover, they are in the limelight because of their accuracy, which is comparable with MMG [25]. Anesthesiologists should increase their knowledge of neuromuscular mechanisms and monitoring, including how to use the latest device for proper neuromuscular monitoring and patient safety.

\section{CONFLICT OF INTEREST}

No potential conflict of interest relevant to this article was reported.

\section{REFERENCES}

1. Duţu M, Ivaşcu R, Tudorache O, Morlova D, Stanca A, Negoiță S, et al. Neuromuscular monitoring: an update. Rom J Anaesth Intensive Care 2018;25:55-60.

2. Thomsen JL, Nielsen CV, Palmqvist DF, Gätke MR. Premature awakening and underuse of neuromuscular monitoring in a registry of patients with butyrylcholinesterase deficiency. Br J Anaesth 2015;115 Suppl 1:i89-i94.

3. Phillips S, Stewart PA, Bilgin AB. A survey of the management of neuromuscular blockade monitoring in Australia and New Zealand. Anaesth Intensive Care 2013;41:374-9.

4. Murphy GS. Neuromuscular monitoring in the perioperative period. Anesth Analg 2018;126:464-8.

5. Loughnan T, Loughnan AJ. Overview of the introduction of neuromuscular monitoring to clinical anaesthesia. Anaesth Intensive Care 2013;41 Suppl 1:19-24.

6. Beecher HK, Todd DP. A study of the deaths associated with anesthesia and surgery: based on a study of 599, 548 anesthesias in ten institutions 1948-1952, inclusive. Ann Surg 1954;140:2-35.

7. Dripps RD, Lamont A, Eckenhoff JE. The role of anesthesia in surgical mortality. JAMA 1961;178:261-6.

8. Thesleff S. An investigation of the muscle-relaxing action of succinyl-choline-iodide in man. Acta Physiol Scand 1952;25:348-67.
9. Christie TH, Churchill-Davidson HC. The St. Thomas's Hospital nerve stimulator in the diagnosis of prolonged apnoea. Lancet 1958;1:776.

10. Churchill-Davidson HC. A portable peripheral nerve-stimulator. Anesthesiology 1965;26:224-6.

11. Roberts DV, Wilson A. Electromyography in the diagnosis and treatment of myasthenia gravis. Br J Pharmacol 1968; 34:229P-30P.

12. Ali HH, Utting JE, Gray C. Stimulus frequency in the detection of neuromuscular block in humans. Br J Anaesth 1970;42:967-78.

13. Ali HH, Utting JE, Gray TC. Quantitative assessment of residual antidepolarizing block. II. Br J Anaesth 1971;43:478-85.

14. Ali HH, Utting JE, Gray TC. Quantitative assessment of residual antidepolarizing block. I. Br J Anaesth 1971;43:473-7.

15. Viby-Mogensen J, Hansen PH, Jørgensen BC, Ording H, Kann T, Fries B. A new nerve stimulator (Myotest). Br J Anaesth 1980;52:547-50.

16. Brull SJ, Kopman AF. Current status of neuromuscular reversal and monitoring: challenges and opportunities. Anesthesiology 2017;126:173-90.

17. Fabregat López J, Candia Arana CA, Castillo Monzón CG. Neuromuscular monitoring and its importance in neuromuscular blockade. Colomb J Anesthesiol 2012;40:293-303.

18. Fuchs-Buder T, Claudius C, Skovgaard LT, Eriksson LI, Mirakhur RK, Viby-Mogensen J; 8th International Neuromuscular Meeting. Good clinical research practice in pharmacodynamic studies of neuromuscular blocking agents II: the Stockholm revision. Acta Anaesthesiol Scand 2007;51:789808.

19. Biro P, Paul G, Dahan A, Brull SJ. Proposal for a revised classification of the depth of neuromuscular block and suggestions for further development in neuromuscular monitoring. Anesth Analg 2019;128:1361-3.

20. Suzuki T, Fukano N, Kitajima O, Saeki S, Ogawa S. Normalization of acceleromyographic train-of-four ratio by baseline value for detecting residual neuromuscular block. Br J Anaesth 2006;96:44-7.

21. Bowdle A, Bussey L, Michaelsen K, Jelacic S, Nair B, Togashi $\mathrm{K}$, et al. A comparison of a prototype electromyograph vs. a mechanomyograph and an acceleromyograph for assessment of neuromuscular blockade. Anaesthesia 2020;75:187-95.

22. Kopman AF. Normalization of the acceleromyographic trainof-four fade ratio. Acta Anaesthesiol Scand 2005;49:1575-6.

23. Liang SS, Stewart PA, Phillips S. An ipsilateral comparison of acceleromyography and electromyography during recovery from nondepolarizing neuromuscular block under general anesthesia in humans. Anesth Analg 2013;117:373-9.

24. Kopman AF, Chin W, Cyriac J. Acceleromyography vs. electromyography: an ipsilateral comparison of the indirectly 
evoked neuromuscular response to train-of-four stimulation. Acta Anaesthesiol Scand 2005;49:316-22.

25. Lee W. The latest trend in neuromuscular monitoring: return of the electromyography. Anesth Pain Med (Seoul) 2021;16:133-7.

26. Bowdle A, Bussey L, Michaelsen K, Jelacic S, Nair B, Togashi K, et al. Counting train-of-four twitch response: comparison of palpation to mechanomyography, acceleromyography, and electromyography. Br J Anaesth 2020;124:712-7.

27. Kazuma S, Wakasugi K, Hagiwara H, Yamakage M. Comparative study of TOF-Cuff, a new neuromuscular blockade monitor, and TOF-watch, an acceleromyography. Anesth Analg 2019;129:e16-9.

28. Krijtenburg P, Honing G, Martini C, Olofsen E, van Elst HJ, Scheffer GJ, et al. Comparison of the TOF-Cuff ${ }^{\circledR}$ monitor with electromyography and acceleromyography during recovery from neuromuscular block. Br J Anaesth 2019;122:e22-4.

29. Eriksson LI. Evidence-based practice and neuromuscular monitoring: it's time for routine quantitative assessment. Anesthesiology 2003;98:1037-9.
30. El-Orbany M. Objective monitoring of neuromuscular block should become the standard of care. Acta Anaesthesiol Scand 2009;53:837.

31. Saager L, Maiese EM, Bash LD, Meyer TA, Minkowitz H, Groudine S, et al. Incidence, risk factors, and consequences of residual neuromuscular block in the United States: the prospective, observational, multicenter RECITE-US study. J Clin Anesth 2019;55:33-41.

32. Nemes R, Renew JR. Clinical practice guideline for the management of neuromuscular blockade: what are the recommendations in the USA and other countries? Curr Anesthesiol Rep 2020;10:90-8.

33. Naguib M, Kopman AF, Ensor JE. Neuromuscular monitoring and postoperative residual curarisation: a meta-analysis. $\mathrm{Br} \mathrm{J}$ Anaesth 2007;98:302-16.

34. Fortier LP, McKeen D, Turner K, de Médicis É, Warriner B, Jones PM, et al. The RECITE study: a canadian prospective, multicenter study of the incidence and severity of residual neuromuscular blockade. Anesth Analg 2015;121:366-72. 